\title{
Avverbi infidi
}

\author{
Ilaria Bonomi
}

PUBBLICATO: 31 LUGLIO 2020

\section{Quesito:}

Con questa scheda rispondiamo a più domande riguardanti le forme peggio e meglio, migliore e peggiore, tanto e poco, anche in combinazione con meno, piu e troppo. Per completare il quadro si rimanda anche alle schede sull'uso di molto migliore, molto maggiore, di troppo pochi e di piú poco in luogo di meno.

\section{Avverbi infidi}

\section{Meglio-peggio per migliore-peggiore}

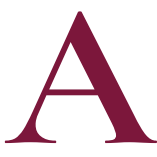

lcuni lettori battono l'accento sull'uso aggettivale degli avverbi comparativi meglio e peggio, in espressioni del tipo "la meglio gioventu" o anche "lei è meglio di suo fratello", criticandolo e sollecitando in merito la voce autorevole dell'Accademia della Crusca.

I due usi devono essere ben distinti, anche se sono accomunati dal valore aggettivale di meglio, la cui funzione principale e più comune è naturalmente quella avverbiale.

Il tipo "lei è meglio di suo fratello", cioè l'uso di meglio come aggettivo comparativo di maggioranza retto da verbi come essere, sembrare, parere, al posto di migliore, è molto comune nella storia dell'italiano e nell'italiano di oggi, e considerato del tutto accettabile da dizionari e grammatiche. Documentato già nei primi grandi autori (per esempio in Dante "fu meglio assai che suo figlio", in Boccaccio "tu sei meglio di lei"), ha avuto una buona vitalità nel corso dei secoli, ed è tuttora vivo e diffuso nella lingua comune e in quella letteraria, senza che ne venga segnalato un carattere "trasgressivo" rispetto alla norma dell'italiano standard.

La stessa cosa possiamo affermare per il corrispondente uso aggettivale di peggio nel senso di peggiore, in un esempio del tipo "la tua idea mi sembra peggio della sua".

Diverso il caso del tipo "la meglio gioventu'" (titolo di una raccolta di poesie di Pasolini, del I954, ripreso poi dal noto film di Marco Tullio Giordana, del 2003), in cui meglio (o peggio) in funzione di aggettivo, preceduto dall'articolo determinativo, prende il valore di superlativo relativo. Si tratta di un uso ben attestato nella storia della letteratura italiana, soprattutto dal '7oo al '9oo, e ben documentato anche ai nostri giorni, come costrutto marcato e caratterizzato in senso regionale, popolare o comunque espressivo e oralizzante. Non a caso lo si trova nel genere epistolare, nella novellistica, nel discorso diretto, e nel teatro. Vediamone qualche esempio: nella "Frusta letteraria" di Giuseppe Baretti "i nostri meglio scrittori", "voglio sapere quali paesi producono il meglio vino, e le meglio cose, e anche le peggio"; nelle Lettere del Carducci "quel pezzo è della meglio prosa che mabbia letto"; nella novella Don Candeloro del Verga "mi rovinano la meglio scena", nel Sor Pietro di Antonio Baldini "le meglio lettere che ho scritto le ho scritte..."; ma anche nella poesia di Montale "il meglio ramicello del tuo orto". Un esempio particolare, proprio nella sottolineatura amaramente ironica del carattere popolare del costrutto, ne fa Pirandello, nella commedia L'innesto: "Ah, quella che è istruzione, signora mia, m'e piaciuta assai, a me, sempre! Non l'ho potuta avere io; ma le mie figliuole, per grazia di Dio, i meglio professori! Francese, inglese, la musica...", o nella novella Donna Mimma "E che hanno da insegnare a me, che li sfascio, li sfascio tutti quanti, i meglio professori". 
Il costrutto ricorre in autori soprattutto toscani, centrali e meridionali, ma non mancano esempi settentrionali come Tommaseo e De Amicis. A questi esempi registrati nel Grande Dizionario della Lingua italiana del Battaglia (GDLI), Google libri aggiunge altre attestazioni, soprattutto da testi romani e napoletani ottocenteschi, e molte da autori di narrativa contemporanea (ricerca fatta per "le meglio cose", "le meglio donne", "le peggio cose").

Il carattere popolare del tipo "la meglio gioventù" nell'italiano comune di oggi è indicato dai dizionari dell'uso: tra questi, il Vocabolario Treccani online precisa "vivo nell'uso popolare di tutte le regioni", e "Anche nell'uso non strettamente popolare o regionale, quando il nome sia taciuto: questi sono i meglio che abbiamo". I medesimi dizionari invece non marcano in alcun modo, considerandolo un uso standard, il tipo "lei è meglio di suo fratello".

Meno precise, in linea generale, sui due costrutti, la loro diffusione, il loro valore e la loro accettabilità sono le grammatiche della lingua italiana. Ne trattano in maniera più puntuale la Grammatica italiana (Italiano nella garzantina) di Luca Serianni (Serianni r989 e 200o), sottolineando del tipo "il meglio vestito, le peggio compagnie" l'uso anche letterario, e il valore prevalentemente popolare, soprattutto ai nostri giorni; analogamente la Nuova grammatica italiana di Maurizio Dardano e Pietro Trifone (Dardano-Trifone I997) ne rileva il valore popolare o regionale oggi, pur esistendone attestazioni letterarie. Insistono, infine, sulla popolarità e la non accettabilità del costrutto Giuseppe Patota e Valeria Della Valle nei loro manualetti normativi (Il salvalingua/Il nuovo salvalingua, Senza neanche un errore, Viva la grammatica, e altri).

Concludendo, dunque, dopo questa breve documentazione della diffusione dei due costrutti in questione, del loro valore e delle indicazioni che su di essi si trovano nei principali strumenti lessicografici e grammaticali, possiamo rispondere sinteticamente così l'uso aggettivale di meglio/peggio retto da essere, sembrare, e simili ("lei è meglio di suo fratello"), è da considerare normale e pienamente accettabile; il tipo, invece "la meglio gioventù", largamente attestato nella storia della lingua italiana e usato da molti e grandi autori, ha carattere popolare, e come tale può, volendo, essere usato in varie occasioni, quando si voglia imprimere al discorso una particolare espressività o una punta di ironia: meglio non usarlo, invece, nella lingua neutra e non caratterizzata della comunicazione, per non correre il rischio di una stigmatizzazione anche sociale, oltre che linguistica.

\section{Meno peggiore e meno peggio sì, più migliore e più meglio no}

Sono ricorrenti anche richieste di delucidazione sulle ragioni dell'accettabilità delle espressioni meno peggio e meno peggiore di contro a piu migliore e piu meglio, talvolta usate ma evidentemente sentite come scorrette: viene rilevato che, essendo la costruzione dei due tipi analoga, con l'avverbio più o meno prima del comparativo, analogo dovrebbe essere il loro valore e la loro accettabilità.

Ma oltre alla componente strettamente grammaticale, o meglio formale, della costruzione, va tenuta in considerazione la componente semantica: per migliore, comparativo organico o sintetico che non ha bisogno dell'avverbio piu per esprimere il valore di maggioranza rispetto all'aggettivo positivo buono, l'aggiunta di piu rappresenterebbe una ridondanza inutile dal punto di vista del significato. Lo stesso vale per il comparativo organico di cattivo, peggiore, e per gli altri comparativi di maggioranza organici maggiore e minore: non si può dire più migliore, più peggiore, più maggiore, più minore (forme che infatti sono state spesso citate tra le caratteristiche devianti dell'italiano popolare). Volendo esprimere un ulteriore grado di comparazione di maggioranza, si usano comunemente gli avverbi molto o ancora: la pizza in questo ristorante é ancora migliore, o questo vino è molto migliore di quell'altro. Con il passare del tempo il valore comparativo di queste forme sintetiche di derivazione latina si è andato perdendo, 
almeno presso parlanti e scriventi meno consapevoli, i quali, considerandoli come aggettivi positivi, li costruiscono con il piu delle forme comparative analitiche del tipo piu buono.

Invece, l'avverbio meno può essere premesso al comparativo di maggioranza peggiore, in quanto indica una riduzione di tale maggioranza: dei due temi mal scritti, il primo é il peggiore, il secondo meno peggiore del primo. Ma davanti a migliore l'avverbio meno suonerebbe contraddittorio, e infatti non si dice meno migliore, e non si dice neanche meno minore e meno maggiore.

Quanto a meglio e peggio, anch'essi non possono essere preceduti da piü; peggio, e non meglio, può essere preceduto da meno: la locuzione sostantivale il meno peggio è molto diffusa, con il significato di "la possibilità, la soluzione, l'evento che, senza essere quelli desiderati o sperati, sono tuttavia da accettare accontentandosi" (Vocabolario Treccani); forse ancora più frequente è la locuzione avverbiale alla meno peggio "in modo veloce e approssimativo" (GRADIT).

Quindi, mentre meno peggiore e meno peggio appartengono alla lingua comune e corretta, piu migliore, più peggiore, più meglio, come anche meno migliore e meno meglio, sono espressioni scorrette.

Sono casi, questi, che dimostrano chiaramente come il funzionamento della lingua dal punto di vista grammaticale abbia come elemento fondamentale anche la componente relativa al significato delle parole e al significato del loro abbinamento in costruzioni sintattiche.

\section{Troppo tanto?}

Anche con questa espressione, sulla cui accettabilità alcuni lettori chiedono lumi, mentre altri chiedono le ragioni per le quali si può dire troppo poco ma non troppo tanto, entriamo nel campo di una trasgressione grammaticale motivata da ragioni semantiche.

Troppo ha tre funzioni grammaticali: avverbio (ho mangiato troppo), aggettivo (ho troppi problemi), pronome (ne ho visti troppi). L'avverbio di quantità troppo "indica eccedenza (o eccessiva scarsità: troppo росо)" (Serianni 1989). Ma si assiste, nell'italiano dei nostri giorni, ad una attenuazione della valenza semantica di eccedenza, in usi esclamativi del tipo "troppo bello!", o "non sto troppo bene", nei quali diventa quasi semanticamente equivalente a molto: nella prima espressione ha una carica espressiva molto maggiore rispetto a molto (infatti è un uso tipico dei giovani), nella seconda è più neutro, ma tipico di un parlato non scelto.

Troppo poco, naturalmente, si può dire, con troppo avverbio di quantità che, premesso a poco, anch'esso avverbio di quantità, indica 'eccessiva scarsità'; normale anche troppo pochi, con l'avverbio troppo (invariabile! cfr. Troppo pochi, non troppi pochi, di Claudio Giovanardi, risposta del 30 novembre 2018 su questo sito) premesso all'aggettivo pochi.

Invece troppo tanto, o troppo tanti non si può dire, perché sarebbe una ridondanza o addirittura una tautologia dal punto di vista del significato: troppo indica già eccedenza, non ha senso premetterlo a tanto. Posso dire, quindi, "ti ho ascoltato troppo poco", ma non "l'ho desiderato troppo tanto", perché basta dire, per lo stesso significato di eccedenza, "l'ho desiderato troppo".

Il costrutto è quindi veramente scorretto e da non usarsi in nessuna situazione, anche se la rete, con la solita ricerca avanzata da Google, ce ne offre qualche esempio, del tutto occasionale. Ma sui dizionari e sulle grammatiche non trova accoglienza, nemmeno per essere messo al bando. 


\section{Cita come:}

Ilaria Bonomi, Avverbi infidi, "Italiano digitale", 2020, XIV, 2020/3 (luglio-settembre) DOI: $10.35948 / 2532-9006 / 2020.4368$

Copyright 2020 Accademia della Crusca

Pubblicato con licenza creative commons CC BY-NC-ND 